\title{
Evaluation of C-shaped Canal Configuration in Maxillary Molars: A Retrospective Cone-Beam Computed Tomography Study
}

\author{
Emre Kose ${ }^{1}$, Ruya Sessiz $A k^{2}$ \\ ${ }^{1}$ Department of Oral and Maxillofacial Radiology, Faculty of Dentistry, Aydın Adnan Menderes University, Aydın, Turkey \\ ${ }^{2}$ Aydın Dental Hospital, Aydın, Turkey \\ Correspondence Author: Ruya Sessiz Ak \\ E-mail: dt.rsessiz@gmail.com
}

Received: $25.08 .2020 \quad$ Accepted: 17.08 .2021

\begin{abstract}
Objective: The aim of this retrospective study was to evaluate the prevalence of C-shaped canal configuration in maxillary first and second molars according to age and gender by using cone beam computed tomography (CBCT) in a Turkish population.

Methods: In this retrospective study, 709 first and 739 second maxillary molars were examined. CBCT images of teeth were examined in different axial sections to determine the presence of C-shaped canal configuration. C-shaped canal configuration was classified into five groups according to the fusion of root canals. Prevalence of $\mathrm{C}$-shaped canal configuration between first and second molars, age groups and genders were compared. The chi-square test was used to compare the categorical variables. The differences were considered significant if the $p$ value was less than 0.05 .

Results: A total of 1448 teeth were evaluated and 3\% $(n=43)$ of them had C-shaped canal configuration. The presence of C-shaped canal configuration was significantly more common in the second molars than the first molars $(p=0.000)$ No significant difference in the prevalence of C-shaped configuration was observed comparing genders and age groups ( $p>0.05)$. Type A and E canal configuration were not detected in first molars.

Conclusion: C-shaped canal configuration is a rare anatomical variation of maxillary molars. This variation includes fins or isthmuses connecting root canals which create challenges during endodontic treatment. Perceiving the variations with $\mathrm{CBCT}$ imaging helps clinicians to understand and treat complex cases without complication. Clinicians should be knowledgeable about the rare variations for better outcomes.

Keywords: Canal Variation, Cbct, C-Shaped Canal, Maxillary Molars
\end{abstract}

\section{INTRODUCTION}

Successful root canal treatment depends on detailed knowledge about canal configuration, the cleaning of all pulp tissues, shaping walls, and the complete obturation of all root canal spaces $(1,2)$. The presence of untreated canals and microorganisms due to inadequate cleaning, shaping, and obturation is the main reason for endodontic treatment failure $(2,3)$. Knowledge of the root and canal variations is one of the most important factors in endodontic procedures in preventing possible complications and in achieving better outcomes $(4,5)$.

Maxillary molars generally have two buccal canals and one palatal canal (6). Maxillary first molars usually show anatomic variation in mesiobuccal root with more than two canals (7). Although the maxillary second molar has a similar morphology to the maxillary first molar, anatomic variations are more common in second molars, such as root fusions, root canal isthmuses, and accessory canals (6). C-shaped canal is another important variation for some teeth, especially for mandibular second molars $(3,5)$. It was first described by Cooke and Cox (1979) as a "ribbon-shaped orifice" (8). Despite a lot of the studies about mandibular molars, there are limited numbers of studies reporting C-shaped canal configurations in maxillary molars. The C-shaped canal configuration can occur in permanent maxillary molars with fused roots and can also continue from the floor of the coronal pulp to the apical region of the root $(3,9)$. This configuration includes grooves, isthmuses, and irregular formations that lead to ineffective cleaning, shaping, and obturation (2).

Periapical radiographs are usually effective for preoperative evaluation of root canal morphology and periapical pathologies (3). These radiographs give 2-dimensional information for 3-dimensional structures. Assessment of root canal in several sections and serial slices is the optimal method for understanding root and canal configurations $(3,10)$. Plain radiographs are insufficient to visualize C-shaped canals because of the canals' complex nature and superimpositions 
(2). Cone beam computed tomography $(C B C T)$ is a tool that gives precise information about root canal complexities. CBCT allows high resolution images in different sections with a small field of view and small voxel size $(4,11)$.

Although the prevalence and types of C-shaped canal configuration in mandibular molars were investigated, there is currently no study on maxillary molars in a Turkish population. The aim of this study is to evaluate the prevalence and types of C-shaped canal configuration in maxillary first and second molars in a Turkish population by using CBCT.

\section{METHODS}

The study protocol was approved by Aydın Adnan Menderes University Faculty of Dentistry Clinical Research Ethics Committee (protocol number: ADUDHF2021/25, date:01.09.2021). For the evaluation of C-shaped canal configuration in maxillary first and second molars, CBCT images taken at our department of oral and maxillofacial radiology for patients' previous dental problems between 2016 and 2019 were examined. Fully erupted and completely root formed maxillary first and second molars without root canal treatment, fracture and crown restoration were included. Third molars and low-quality images were excluded. Finally, 1448 maxillary molars (709 first molars and 739 second molars) in 517 Turkish patients were examined retrospectively.

The CBCT scans were obtained using standard exposure parameters and patient positioning protocols (resolution: voxel size $0.2 \mathrm{~mm}, 80-84 \mathrm{kvp}, 10-12 \mathrm{~mA}$ ) with a $\mathrm{CBCT}$ unit (Planmeca Promax 3D Max; Helsinki, Finland). Romexis version 4.6.2. software (Planmeca; Helsinki, Finland) was used for evaluation of axial, sagittal, and coronal CBCT planes with optimal contrast and brightness adjustment. Images were examined in a 22-inch LCD monitor (Samsung Business Monitor S22D300HY; UK) with a resolution of 1,920 × 1,080 pixels in a dark room. For the proper assessment, axial sections that were vertical to the long axis of each tooth were examined by rolling the tool bar from the floor of coronal pulp to the apex. All images were evaluated retrospectively by two oral and maxillofacial radiologists with at least two years' experience in performing CBCT. The observers evaluated the images twice in one-month intervals to assess intra - and interexaminer agreement.

Five groups (10-22, 23-36, 37-50, 51-64, 65-77) were created according to age range. Because no well-defined classification could be determined in the case of C-shaped canals in maxillary molars, modified classification of a lower molar C-shaped canal was used according to Martins et al. (5) as below:

The canal system was evaluated at 5 axial levels:

1. $2 \mathrm{~mm}$ below the canal beginning

2. Middle interval between coronal and middle
3. Middle interval from the canal beginning and anatomic apex

4. Middle interval between the middle and apical

5. $2 \mathrm{~mm}$ above the anatomic apex

Canal systems continuous at 3 axial levels and large C-shaped canal systems with 2 main canal lumens connected by a large isthmus were accepted as a C-shaped canal. C-shaped maxillary molars according to the configuration of the fused root canals were classified (Figure 1, 2).

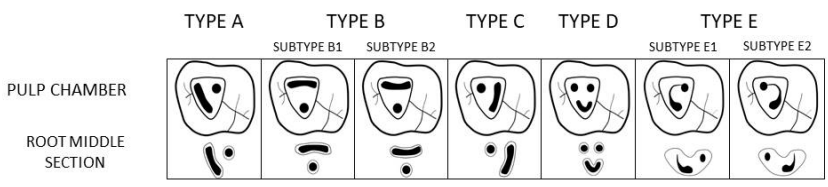

Figure 1. Schematic drawings of C-shaped canal configurations according to root canal fusion

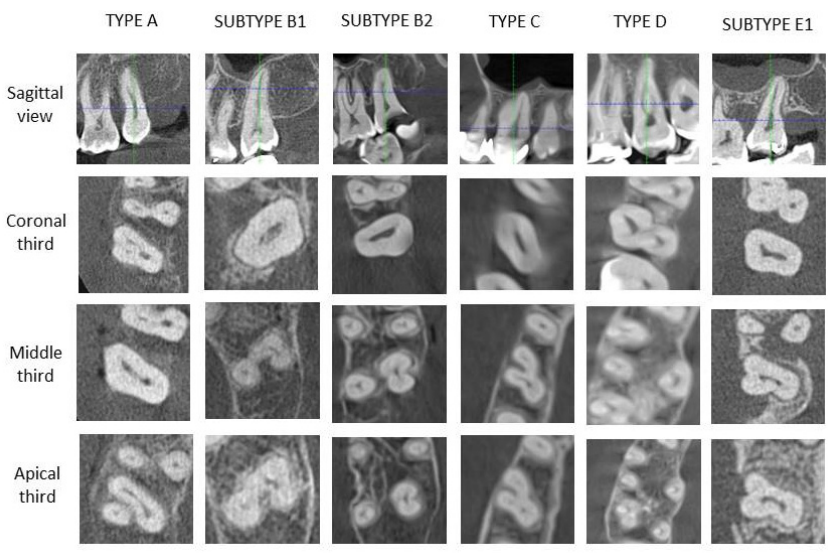

Figure 2. Axial views of each types of C-shaped canal configurations

\subsection{Statistical Analysis}

All the data were analyzed with the SPSS software (IBM SPSS Statistics Version 22; IBM, Armonk, New York). The chisquare test was used to compare the prevalence of C-shaped canals in first and second molars across genders, age groups, and sides. When the $p$ value was $<0.05$, the differences were considered significant. Inter - and intraobserver reliability was calculated using the Cohen kappa test.

\section{RESULTS}

A total of 517 patients ( 231 men and 286 women) CBCT images including 709 maxillary first molars and 739 maxillary second molars were examined in this study. Mean age of the patients was 34.2 years, ranging from 10 to 77 years. Three percent (43) of all teeth had C-shaped root canals, and 2.9\% (24) of women and 3\% (19) of men had C-shaped canal in their maxillary molars. There was no significant difference between the genders $(p=0.876)$. 
Sides and numbers of maxillary molars with the C-shaped canal configuration were presented in Table 1 . Nineteen (2.6\%) right maxillary molars and 24 (3.3\%) left maxillary molars had a C-shaped canal. There was no significant difference between sides ( $p=0.423)$. The $C$-shape root canal system was more common in maxillary second molars than maxillary first molars with ratios $4.9 \%$ and $1 \%$, respectively. The difference between the first and second maxillary molars was considered significant $(p=0.000)$.

Table 1. C-shaped canal configuration in maxillary first and second molars

\begin{tabular}{|c|c|c|c|c|}
\hline & \multicolumn{4}{|c|}{$\begin{array}{l}\text { Right Maxillary Molars } \\
\text { Left Maxillary Molars }\end{array}$} \\
\hline & First & Second & First & Second \\
\hline Present & 3 & 16 & 4 & 20 \\
\hline Absent & 355 & 353 & 347 & 350 \\
\hline \multirow{2}{*}{ Total } & 358 & 369 & 351 & 370 \\
\hline & \multicolumn{2}{|l|}{727} & 721 & \\
\hline
\end{tabular}

Types of C-shaped canal configuration according to first and second molars were presented in Table 2. Type $A$ and $E$ were not found in first molars. The most common type was type $B$, with a prevalence of $2.3 \%$ in maxillary second molars. There was no significant difference between types of C-shaped canals ( $p=0.563$ ). Prevalence of $C$-shaped canal configuration according to age groups was presented in Table 3. There was no significant relationship between the presence of C-shaped canal configuration and age groups.

Table 2. Types of $C$ shaped canals in maxillary first and second molars

\begin{tabular}{|c|c|c|c|c|c|c|c|c|c|}
\hline & & \multicolumn{4}{|c|}{ Maxillary First Molars } & \multicolumn{4}{|c|}{ Maxillary Second Molars } \\
\hline & & Right & Left & Total & Per cent & Right & Left & Total & Per cent \\
\hline \multirow{6}{*}{ 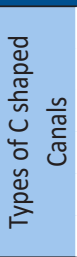 } & A & 0 & 0 & 0 & $0 \%$ & 3 & 1 & 4 & $0.5 \%$ \\
\hline & B & 1 & 1 & 2 & $0.3 \%$ & 8 & 9 & 17 & $2.3 \%$ \\
\hline & C & 1 & 1 & 2 & $0.3 \%$ & 1 & 4 & 5 & $0.7 \%$ \\
\hline & D & 1 & 2 & 3 & $0.4 \%$ & 1 & 1 & 2 & $0.2 \%$ \\
\hline & $E$ & 0 & 0 & 0 & $0 \%$ & 3 & 5 & 8 & $1.1 \%$ \\
\hline & Total & 3 & 4 & 7 & $1 \%$ & 16 & 20 & 36 & $4.8 \%$ \\
\hline
\end{tabular}

Table 3. Prevelance of $\mathrm{C}$-shaped canal configuration in age groups

\begin{tabular}{|c|c|c|c|c|}
\hline \multirow[b]{2}{*}{ Age Groups } & \multirow[b]{2}{*}{ Maxillary molars } & \multicolumn{2}{|c|}{$\begin{array}{l}\text { C-shaped canal } \\
\text { configuration }\end{array}$} & \multirow[b]{2}{*}{ TOTAL } \\
\hline & & Present & Absent & \\
\hline \multirow{2}{*}{$10-22$} & First molar & 2 & 297 & \multirow{2}{*}{591} \\
\hline & Second molar & 7 & 285 & \\
\hline \multirow{2}{*}{$23-36$} & First molar & 2 & 169 & \multirow{2}{*}{338} \\
\hline & Second molar & 10 & 157 & \\
\hline \multirow{2}{*}{$37-50$} & First molar & 5 & 157 & \multirow{2}{*}{329} \\
\hline & Second molar & 11 & 156 & \\
\hline \multirow{2}{*}{$51-64$} & First molar & 1 & 86 & \multirow{2}{*}{172} \\
\hline & Second molar & 4 & 81 & \\
\hline \multirow{2}{*}{$65-77$} & First molar & 0 & 8 & \multirow{2}{*}{18} \\
\hline & Second molar & 1 & 9 & \\
\hline TOTAL & & 43 & 1405 & 1448 \\
\hline
\end{tabular}

The relative technical error of measurements for intra - and interobserver errors ranged from 1.02 to $1.62 \%$ and from 1.1 to $1.94 \%$, respectively. The coefficient of reliability values ranged from 0.94 to 0.98 .

\section{DISCUSSION}

Several imaging techniques and demineralization/staining methods have been used to visualize detailed root canal morphology in the literature (12-16). Periapical radiographs, examination of root canals during endodontic treatment, patients' previous records, and CBCT are frequently used in clinical studies of root canal configuration $(1,17-19)$. Most of these studies have used Vertucci's classification system for root canal identification (13). Unlike previous studies, we classified root canals according to their axial views. Also, this study used CBCT images to investigate the presence of C-shaped canals according to gender, age, and location.

In clinical practice, C-shaped canal configuration can be assessed with periapical radiographs, CBCT and CT (2023). СBCT has many advantages, such as lower radiation doses, lower exposure time, higher resolution, lower voxel sizes, and more precise measurements. СBCT also allows cross-sectional images without superimposition, which is not possible with intraoral radiographs $(24,25)$. Because of the radiation dose, SEDENTEXCT guidelines (26). In our study, CBCT images that were taken of the patient's previous dental problems, such as intrabony cysts, implant planning, and orthodontic problems, were evaluated retrospectively.

There were a limited numbers of studies that evaluated C-shaped canals in maxillary molars $(1,5,17)$. Most studies on C-shaped canals were focused on mandibular molars due to their predisposition to this variation $(8,15,16)$. We could not find any reported study in the literature about C-shaped canal configuration in maxillary molars in the Turkish population and highlighted the importance of this variation for clinical practice. In our study, the prevalence of C-shaped canals in maxillary first molars and second molars was $1 \%$ and $4.9 \%$, respectively. Similarly, Yang et al. reported a $4.9 \%$ prevalence for maxillary second molars by using a clearing method in a Chinese population study (27). In a Korean population study using $\mathrm{CBCT}$, the prevalence of C-shaped root canals in maxillary first molars and second molars was $0.8 \%$ and $2.7 \%$, respectively (1). Compared with the present study, our result was lower in the first molars and higher in the second molars. In our study, maxillary second molars were more prone to have C-shaped canals than first molars. In the study of De Moor et al. (17), which was performed on radiographs, reported a lower prevalence $(0.091 \%)$ than our result in maxillary first molars. The difference may be due to the method of study and ethnic background. Other differences from the previous studies may be due to subject age, study size, imaging types, and racial features.

With the exception of type $D$, all other types of C-shaped canals were more common in second molars. In contradistinction 
to our results, in the Martin et al. study only type $C$ was more common in first molars. The second most common type was type $\mathrm{E}$ in second molars with $1.1 \%$ prevalence and a deep isthmus and groove. These areas can go undetected clinically, allowing pathogen microorganisms to colonize easily, resulting in failed endodontic treatment. Because of a complex internal configuration, the Type E canal poses more difficulties in root canal treatment compared with Type C. Our results were similar to those of Martin et al., who did not find Types $D$ and $E$, and we did not find types $A$ and $E$ in first molars. Martin et al. found Type $C$ with a prevalence of $0.2 \%$ in second molars and $0.9 \%$ in first molars. In our study, Type $\mathrm{C}$ had a prevalence of $0.7 \%$ in second molars and $0.3 \%$ in first molars.

From the previous studies, only Martin et al. (5) investigated C-shaped canal configuration according to sex and side. In their study, a C-shaped canal was more common in females and not related to the right or left side (5). Similarly, there was no significant difference between the right and left side in our study. Most clinical cases of C-shaped canal configuration in maxillary molars were presented in males $(2,7,28)$. A previous case report on $\mathrm{C}$-shaped canal configuration in maxillary second molars was presented in a woman with spiral CT(3). In our study, C-shaped canals were more common in females without significant differences (29). The C-shaped canal configuration can gradually disappear due to secondary and tertiary dentine formation during aging. In the literature there is no explicit relationship between age and prevalence of C-shaped canal configuration in maxillary molars. Despite the low prevalence ratio of this variation in our study, we divided patients into age groups to compare possible changes. But we did not find any relationship between age and presence of the C-shaped canal configuration in maxillary molars.

In the literature, the C-shaped canal configuration of maxillary molars was described according to fused roots and only serial sections of axial view $(1,5)$. There has not been any well-documented or accepted classification for maxillary molars yet. Evaluation of root canal morphology has been done with various methods both in vivo and in vitro $(1,4$, $30,31)$. There were some limitations in this study. First of all, only an Aegean region population was included in our study. In future studies, patients from all over the country should be included in the study. In future studies, patients from other regions of the world with larger populations should be included. Secondly, root forms and root dentin thickness were not examined in the present study. It would be helpful for instrumentation and obturation of root canals.

\section{CONCLUSION}

This retrospective CBCT study of a Turkish population showed a low prevalence of C-shaped canal configuration in maxillary molars. Results of future studies including different populations, large samples, and different highresolution imaging methods may help to elucidate this variation. If the clinician suspects the presence of C-shaped canal configuration in maxillary molar with root fusion, a high-resolution CBCT scan should be performed and examined carefully to prevent endodontic failures.

\section{REFERENCES}

[6] Jo HH, Min JB, Hwang HK. Analysis of C-shaped root canal configuration in maxillary molars in a Korean population using cone-beam computed tomography. Restor Dent Endod. 2016;41(1):55-62.

[7] Kottoor J, Velmurugan N, Ballal S, Roy A. Four-rooted maxillary first molar having C-shaped palatal root canal morphology evaluated using cone-beam computerized tomography: a case report. Oral Surg Oral Med Oral Pathol Oral Radiol Endod. 2011;111(5):41-45.

[8] Singla M, Aggarwal V. C-shaped palatal canal in maxillary second molar mimicking two palatal canals diagnosed with the aid of spiral computerized tomography. Oral Surg Oral Med Oral Pathol Oral Radiol Endod. 2010;109(6):92-95.

[9] Bulut DG, Kose E, Ozcan G, Sekerci AE, Canger EM, Sisman Y. Evaluation of root morphology and root canal configuration of premolars in the Turkish individuals using cone beam computed tomography. Eur. J. Dent.2015;9(4):551.

[10] Martins JN, Mata A, Marques D, Anderson C, Caramês J. Prevalence and characteristics of the maxillary C-shaped molar. J Endod. 2016;42(3):383-389.

[11] Ordinola-Zapata R, Martins J, Bramante CM, Villas-Boas M, Duarte $M$, Versiani M. Morphological evaluation of maxillary second molars with fused roots: a micro-CT study. Int. Endod. J. 2017;50(12):1192-1200.

[12] Martins JN, Quaresma S, Quaresma MC, Frisbie-Teel J. C-shaped maxillary permanent first molar: a case report and literature review. J Endod. 2013;39(12):1649-1653.

[13] Cooke HG, Cox FL. C-shaped canal configurations in mandibular molars. J Am Dent Assoc. 1979;99(5):836-839.

[14] Lopes DS, Câmara AC, Aguiar CM, Do Nascimento MDCC, De Araujo LF. A C-Shaped Canal in a Maxillary Second Molar: Prexion 3D Cone-Beam Computed Tomography Analysis. Acta Stomatol. Croat. 2016;50(4):354.

[15] Reddy YP, Karpagavinayagam K, Subbarao C. Management of dens invaginatus diagnosed by spiral computed tomography: a case report. J Endod. 2008;34(9):1138-1142.

[16] Patel S, Durack C, Abella F, Roig M, Shemesh H, Lambrechts P. European Society of Endodontology position statement: the use of CBCT in endodontics. Int. Endod. J. 2014;47(6):502-504.

[17] Neelakantan P, Subbarao C, Ahuja R, Subbarao CV. Root and canal morphology of Indian maxillary premolars by a modified root canal staining technique. Odontology. 2011;99(1):18-21.

[18] Sert S, Bayirli GS. Evaluation of the root canal configurations of the mandibular and maxillary permanent teeth by gender in the Turkish population. J Endod. 2004;30(6):391-398.

[19] Weng X-L, Yu S-B, Zhao S-L, Wang H-G, Mu T, Tang R-Y. Root canal morphology of permanent maxillary teeth in the Han nationality in Chinese Guanzhong area: a new modified root canal staining technique. J Endod. 2009;35(5):651-656.

[20] Lambrianidis T, Lyroudia K, Pandelidou O, Nicolaou A. Evaluation of periapical radiographs in the recognition of C-shaped mandibular second molars. Int. Endod. J. 2001;34(6):458-62.

[21] Fan B, Cheung GS, Fan M, Gutmann JL, Bian Z. C-shaped canal system in mandibular second molars: part I-anatomical features. J Endod. 2004;30(12):899-903. 
[22] De Moor R. C-shaped root canal configuration in maxillary first molars. Int. Endod. J. 2002;35(2):200-208.

[23] Ross IF, Evanchik PA. Root fusion in molars: incidence and sex linkage. J. Periodontol. 1981;52(11):663-667.

[24] Trope M, Elfenbein L, Tronstad L. Mandibular premolars with more than one root canal in different race groups. J Endod. $1986 ; 12(8): 343-345$.

[25] Zheng Q-h, Wang Y, Zhou X-d, Wang Q, Zheng GN, Huang DM. A cone-beam computed tomography study of maxillary first permanent molar root and canal morphology in a Chinese population. J Endod. 2010;36(9):1480-1484.

[26] Neelakantan P, Subbarao C, Ahuja R, Subbarao CV, Gutmann JL. Cone-beam computed tomography study of root and canal morphology of maxillary first and second molars in an Indian population. J Endod. 2010;36(10):1622-1627.

[27] Zhang R, Yang $H$, Yu X, Wang $H, H u ~ T$, Dummer PMH. Use of CBCT to identify the morphology of maxillary permanent molar teeth in a Chinese subpopulation. Int. Endod. J. 2011;44(2):162-169.

[28] Kim Y, Lee SJ, Woo J. Morphology of maxillary first and second molars analyzed by cone-beam computed tomography in a Korean population: variations in the number of roots and canals and the incidence of fusion. J Endod. 2012;38(8):10631068.

[29] Lascala C, Panella J, Marques MM. Analysis of the accuracy of linear measurements obtained by cone beam computed tomography (CBCT-NewTom). DENTOMAXILLOFAC RAD. 2004;33(5):291-294.

[30] Cassetta M, Stefanelli LV, Pacifici A, Pacifici L, Barbato E. How accurate is CBCT in measuring bone density? A comparative CBCT-CT in vitro study. CLIN IMPLANT DENT R. 2014;16(4):471478.

[31] Horner K. Radiation protection: cone beam CT for dental and maxillofacial radiology provisional guidelines 2009. SedentextCT; 2009.

[32] Yang ZP, Yang SF, Lee G. The root and root canal anatomy of maxillary molars in a Chinese population. Dent Traumatol. 1988;4(5):215-218.

[33] Newton CW, McDonald S. A C-shaped canal configuration in a maxillary first molar. J Endod. 1984;10(8):397-399.

[34] Helvacioglu-Yigit D, Sinanoglu A. Use of cone-beam computed tomography to evaluate $\mathrm{C}$-shaped root canal systems in mandibular second molars in a Turkish subpopulation: a retrospective study. Int Endod J. 2013;46(11):1032-1038.

[35] Pawar AM, Pawar M, Kfir A, Singh S, Salve P, Thakur B. Root canal morphology and variations in mandibular second molar teeth of an Indian population: an in vivo cone-beam computed tomography analysis. Clin. Oral Investig. 2017;21(9):28012809.

[36] Manning S. Root canal anatomy of mandibular second molars: Part II C-shaped canals. Int. Endod. J. 1990;23(1):40-45.

How to cite this article: Kose E, Sessiz Ak R. The Relationship Between Evaluation of C-shaped Canal Configuration in Maxillary Molars: A Retrospective Cone-Beam Computed Tomography Study. Clin Exp Health Sci 2021; 11: 444-448. DOI: 10.33808/ clinexphealthsci.785232 